Archive for

Organic Chemistry

Arkivoc 2019, part vi, 180-189

\title{
Acid-catalyzed reaction of 1-(2,2-dimethoxyethyl)ureas with phenols as an effective approach to diarylethanes and dibenzoxanthenes
}

A. V. Smolobochkin, ${ }^{a}$ A. S. Gazizov, ${ }^{a}$ J. K. Voronina, ${ }^{b, c}{ }^{\text {T.S. Rizbayeva, }}{ }^{\text {a A. R. Burilov, }}{ }^{a}$ and M. A. Pudovik ${ }^{a}$

${ }^{a}$ Arbuzov Institute of Organic and Physical Chemistry, FRC Kazan Scientific Center, Russian Academy of Sciences, Arbuzova str., 8, Kazan, Russian Federation

${ }^{b}$ N.S. Kurnakov Institute of General and Inorganic Chemistry, Russian Academy of Sciences, 31 Leninsky Ave., Moscow, Russian Federation

${ }^{c}$ A. N. Nesmeyanov Institute of Organoelement Compounds of Russian Academy of Sciences, Vavilova Str. 28, 119991 Moscow, Russian Federation

Email: smolobochkin@iopc.ru

Received 09-04-2019

Accepted 09-29-2019

Published on line 11-07-2019

\section{Abstract}

A one-pot synthesis of urea-substituted diarylethanes and dibenzoxanthenes starting from 1-(2,2dimethoxyethyl)ureas and phenols has been developed. The approach uses readily available reagents and catalysts, requires mild reaction conditions and provides the target compounds in good to high yields.

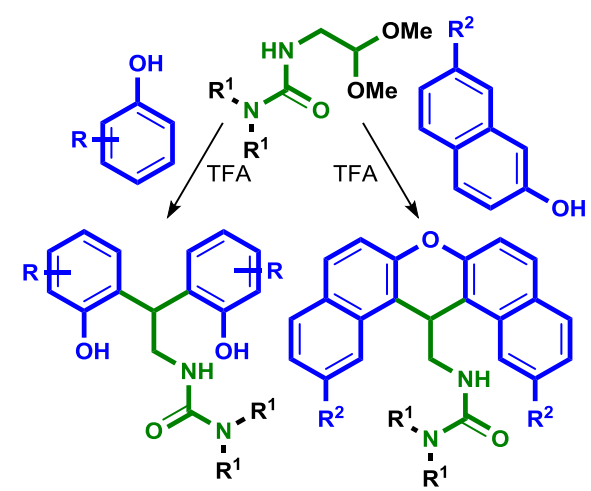

Keywords: Phenols, dibenzoxanthenes, diarylethanes, acetals 


\section{Introduction}

Diarylethanes containing urea fragments are known for their pharmacological activity. According to the literature, these compounds exhibit antibacterial activity, ${ }^{1-3}$ may act as inhibitors of lysine-specific histone demethylase 1 (LSD1) $)^{4}$ and antagonists of muscarinic acetylcholine ${ }^{5}$ and chemokine type 3 receptors (CCR3) . $^{6}$. Additionally, some of them can be used in malaria ${ }^{7}$ and sclerosis $^{8}$ treatment (Figure $\left.1, A\right)$. The main synthetic approach to urea-containing diarylethanes is based on the reaction of amines with isocyanates (Figure 1, B). While from a technical point of view the approach is simple, it suffers from hard to access starting amines and/or isocyanates. As a result, varying the target urea substituents is a complex and cumbersome task.
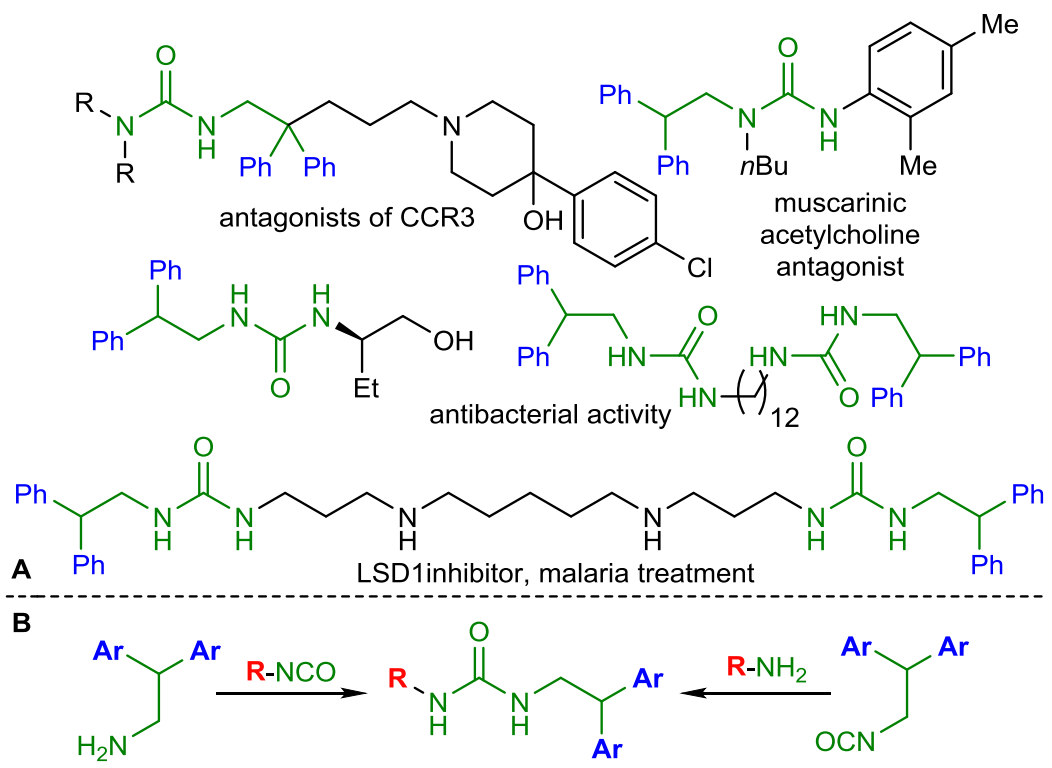

Figure 1. A: Diarylethane-based ureas of biological importance. B: Synthetic approach to urea-containing diarylethanes.

At the same time, the chemistry of nitrogen-containing acetals is gaining increased interest, as indicated by a number of recent review papers. ${ }^{9-12}$ The main reason for this, is the presence of both nucleophilic nitrogen and electrophilic $\alpha$-carbon atoms leading to a diverse array of potential reactions. Moreover, nitrogen-containing acetals serve as valuable reagents for the synthesis of various biologically active compounds, both natural and synthetic. For example, Erythroxylon coca alkaloids were obtained with high enantioselectivity via acid-catalyzed cyclization of 2,2'-\{[1,9-di(1,3-dioxan-2-yl)nonane-3,7diyl]bis(azanediyl)\}bis(2-phenylethan-1-ol). ${ }^{13}$ 1-[2-(1,3-Dioxolan-2-yl)ethyl]-6,7-dimethoxy-1,2,3,4-tetrahydroisoquinoline was used as starting compound in the synthesis of $S$-(-)-crispin $A .{ }^{14,15}$ Similarly, $N-(2,2-$ methoxyethyl)- $1 \mathrm{H}$-indole-2-carboxamide was employed in the synthesis of carbolines. ${ }^{16}$ Kuehne et al. $^{17}$ described a synthesis of 18-methoxycoronaridine from methyl 5-[1-(1,3-dioxolan-2-yl)-3-methoxypropyl]1,2,3,4,5,6,7,8-octahydroazonino[5,4- $b$ ] indole-7-carboxylate. 2,2-Dimethoxyethan-1-amine is used in one stage of the synthesis of the anthelmintic drug praziquantel. ${ }^{18}$ Recently, we successfully used $\mathrm{N}$-substituted 4,4-diethoxybutane-1-amines to prepare pyrazolyl-substituted nicotine analogues. ${ }^{19}$

Herein, we describe results obtained during our ongoing research of nitrogen-containing acetal chemistry and report the employment of 2,2-dimethoxyethane-1-amines in the synthesis of urea-containing diarylethanes and dibenzoxanthenes. 


\section{Results and Discussion}

Previously, we reported a synthesis of imidazolidin-2-ones via acid-catalyzed reaction of 1-(2,2dimethoxyethyl)-3-arylurea with resorcinol and its derivatives. ${ }^{20-22}$ According to the proposed mechanism, the key factor affecting a new $\mathrm{C}-\mathrm{N}$ bond formation and intramolecular heterocyclic ring closure is a presence of the $\mathrm{NH}$ group in the molecule of the starting urea. We speculated that a presence of a second substituent at the nitrogen atom will prevent intramolecular cyclization of 1-(2,2-dimethoxyethyl)ureas and a reaction will result in a formation of acyclic diarylethanes. Not only would this allow a one-step synthesis of target compounds, but also the easy availability of starting compounds would be advantageous. With this in mind, we obtained starting acetals 2 via the reaction of 2,2-dimethoxyethane-1-amine 1 with 1,1-disubstituted carbamoyl chlorides (Scheme 1). Both $N, N$-dialkyl- and $N, N$-diaryl-substituted ureas were obtained to check whether the nature of a substituent affects a reaction coarse.

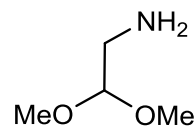

1

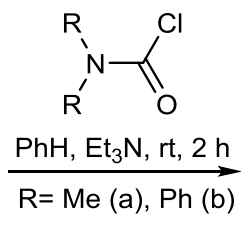

OMe

$2 a, b$

Scheme 1. Synthesis of acetals $2 \mathbf{a}, \mathbf{b}$.

Next, we carried out the reaction of the synthesized acetals $\mathbf{2} \mathbf{a}, \mathbf{b}$ with 4 -chlororesorcinol and sesamol. The reaction proceeded smoothly at room temperature in the presence of trifluoroacetic acid and resulted in the diarylethanes $\mathbf{3 a , b}$ with fairly good yield (Table 1, entries 1 and 2). Earlier, we showed that reactions of nitrogen-containing acetals can also be used to prepare dibenzoxanthenes. ${ }^{23-25}$ Thus, 2-naphthol and 2,7naphthalenediol were used in the reaction with acetals $\mathbf{2} \mathbf{a}, \mathbf{b}$ in the presence of an excess of trifluoroacetic acid (Scheme 2). As a result, dibenzoxanthenes 4a-d were obtained in good yields (Table 1, entries 3-6). Notably, no influence of substituents at the nitrogen atom on target compound yields was detected (cf. Table 1, entries $3,5$ and 4,6$)$.<smiles>[R]C([R])CNC(=O)N([R])[R]</smiles>

3a,b

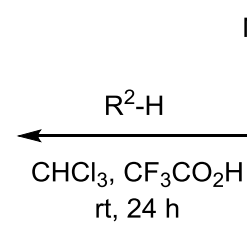

rt, $24 \mathrm{~h}$

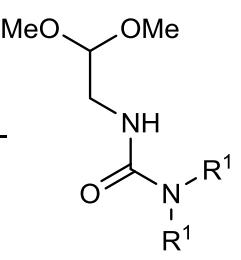

2a,b

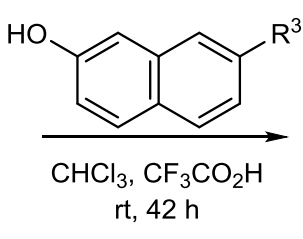

$\mathrm{rt}, 42 \mathrm{~h}$

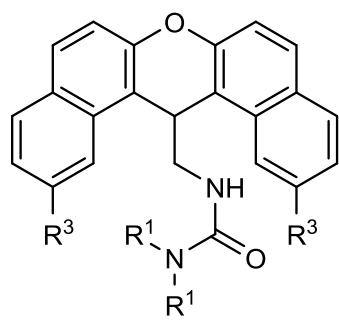

4a $R^{1}=M e, R^{3}=H$

4b $\mathrm{R}^{1}=\mathrm{Me}, \mathrm{R}^{3}=\mathrm{OH}$

4c $\mathrm{R}^{1}=\mathrm{Ph}, \mathrm{R}^{3}=\mathrm{H}$

$4 d R^{1}=P h, R^{3}=O H$

Scheme 2. Synthesis of diarylethanes $\mathbf{3}$ and dibenzoxanthenes 4 . 
Table 1. Synthesis of diarylethanes $\mathbf{3 a , b}$ and dibenzoxanthenes $4 \mathbf{a}-\mathbf{d}$

\begin{tabular}{cccrccc}
\hline № & Acetal & $\mathrm{R}^{1}$ & $\mathrm{R}^{2}$ & $\mathrm{R}^{3}$ & Product & Yield (\%) \\
\hline 1 & $\mathbf{2 a}$ & $\mathrm{Me}$ & 5-chloro-2,4-dihydroxyphenyl & - & $\mathbf{3 a}$ & 66 \\
$\mathbf{2}$ & $\mathbf{2 b}$ & $\mathrm{Ph}$ & 6-hydroxybenzo[d][1,3]dioxol-5-yl & - & $\mathbf{3 b}$ & 88 \\
3 & $\mathbf{2 a}$ & $\mathrm{Me}$ & - & $\mathrm{H}$ & $\mathbf{4 a}$ & 87 \\
4 & $\mathbf{2 a}$ & $\mathrm{Me}$ & - & $\mathrm{OH}$ & $\mathbf{4 b}$ & 70 \\
5 & $\mathbf{2 b}$ & $\mathrm{Ph}$ & - & $\mathrm{H}$ & $\mathbf{4 c}$ & 79 \\
6 & $\mathbf{2 b}$ & $\mathrm{Ph}$ & - & $\mathrm{OH}$ & $\mathbf{4 d}$ & 68 \\
\hline
\end{tabular}

${ }^{a}$ Isolated yield.

The bond lengths, valence and torsion angles taken from a single crystal $\mathbf{x}$-ray structure of compound $\mathbf{4 c}$ were in the ranges typical for every bond type as proved by X-ray analysis. The conformation of molecule $\mathbf{4 c}$ is folded and stabilized by $\mathrm{CH}(\mathrm{Ph}) . . . \pi$ and $\pi . . . \pi$ interactions (Figure 1 ).

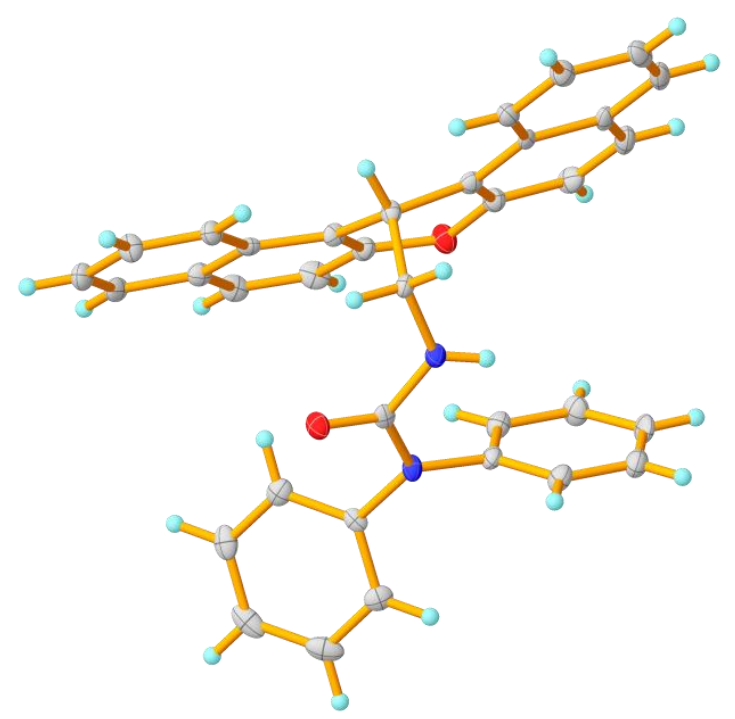

Figure 1 Molecular structure of compound $4 \mathrm{c}$ in the crystalline state. Ellipsoids are shown with $50 \%$ probability.

The crystal packing of compound $\mathbf{4 c}$ involves a large number of non-covalent interactions. Infinite chains are formed by the hydrogen bonds with additional stabilization of $L p . . . \pi$ interactions. Folded layers are formed perpendicular to them due to stacking and $\mathrm{CH}$... $\pi$ interactions (Figure 2).

The proposed reaction mechanism is similar to that previously described for formation of polyphenols from $N$-(4,4-diethoxybutyl)sulfonylamides (Scheme 3). ${ }^{24}$ The key step of the reaction is the elimination of methanol from acetal $\mathbf{2}$, which affords oxonium ion $\mathbf{A}$. The next stage is the interaction of this species with phenolic nucleophile. Depending on the phenol used, the reaction can lead either to dibenzoxanthenes 4 or diarylethanes $\mathbf{3}$. The use of naphthol leads to an intermediate compound $\mathbf{B}$; and subsequent stages result in dibenzoxanthenes 4 according to the previously described mechanism. ${ }^{24}$ When 4 -bromoresorcinol or sesamol are used as nucleophiles, diarylethanes $\mathbf{3}$ are formed through the intermediate $\mathbf{E}$ and benzylic cation $\mathbf{F}$. 


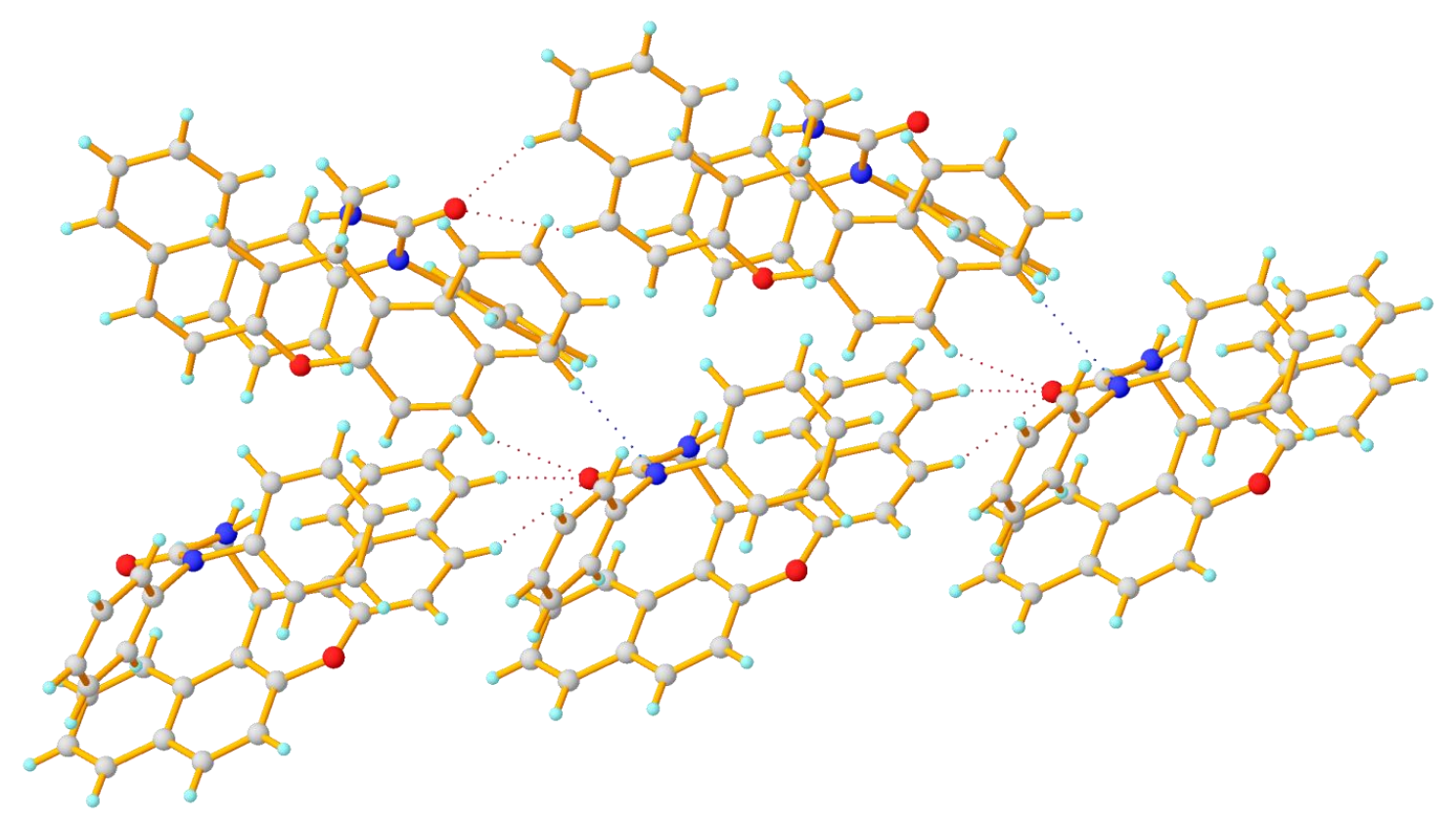

Figure 2 Fragment of H-bonded chain in crystal packing of compound 4c.

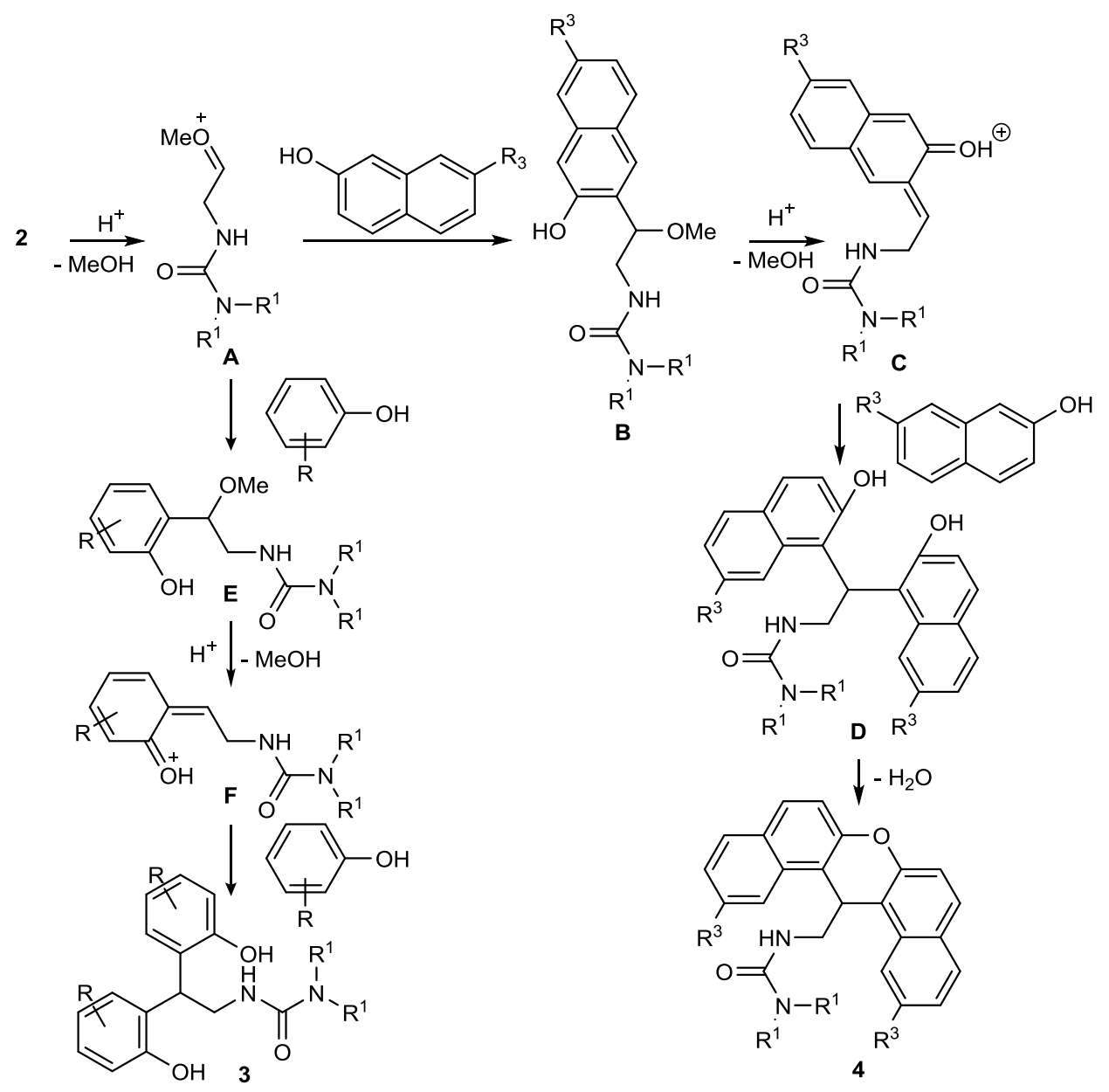

Scheme 3. Proposed mechanism for the substituted ureas $\mathbf{3 , 4}$ formation. 


\section{Conclusions}

In conclusion, we have developed a convenient one-pot protocol for the synthesis of otherwise hard to access diarylethanes and dibenzoxanthenes via metal-free acid-catalyzed reaction of phenols with 1-(2,2dimethoxyethyl)ureas.

\section{Experimental Section}

General. ${ }^{1} \mathrm{H}$ NMR spectra were recorded on Bruker MSL 400 spectrometer (working frequency $400.13 \mathrm{MHz}$ ) in $\mathrm{CDCl}_{3}$ and $\left(\mathrm{CD}_{3}\right)_{2} \mathrm{SO}$ relative to the residual solvent protons. ${ }^{13} \mathrm{C}$ NMR spectra were recorded on Bruker Avance 600 spectrometer (working frequency $150.90 \mathrm{MHz}$ ). The MALDI-TOF mass spectra were recorded on a Bruker ULTRAFLEX III TOF/TOF instrument (with 2,5-dihydroxybenzoic acid matrix). IR spectra were obtained with a Bruker Vector 22 spectrometer. Elemental analysis was performed on Carlo Erba EA 1108 instrument. Schöniger method was applied for chlorine determination. ${ }^{28}$ Melting points were determined in glass capillaries with a Stuart SMP 10 apparatus. Single-crystal X-ray diffraction analysis was performed at 295(2) K on Smart Apex II automatic diffractometer using graphite monochromated radiation. All solvents were purified and dried according to standard procedures.

The X-ray diffraction data for the crystal of $\mathbf{4 c}$ were collected on a Smart Apex II automatic diffractometer using graphite monochromated radiation. The structures were solved by direct methods and refined by fullmatrix least-squares using the SHELXL97 ${ }^{26}$ program. All the non-hydrogen atoms were refined with anisotropic atomic displacement parameters. All figures were made using the program OLEX2 ${ }^{27}$. Crystallographic data for the structure reported in this paper have been deposited with the Cambridge Crystallographic Data Center (deposit number is 1950456).

General method for the synthesis of ureas $\mathbf{2 a , b}$. To a solution of 2,2-dimethoxyethan-1-amine $(0.90 \mathrm{~g}, 8.60$ $\mathrm{mmol}$ ) and triethylamine $(1.74 \mathrm{~g}, 17.21 \mathrm{mmol})$ in benzene $(15 \mathrm{~mL})$ was added dropwise with cooling $\left(5-7^{\circ} \mathrm{C}\right)$, carbamoyl chloride $(8.60 \mathrm{mmol})$. The reaction mixture was stirred under cooling for 2 hours. The precipitate was filtered off, the filtrate was evaporated in vacuum to afford the oily product.

3-(2,2-Dimethoxyethyl)-1,1-dimethylurea (2a). Yellow oil, yield $1.35 \mathrm{~g}, 89 \% .{ }^{1} \mathrm{H} N M R\left(\mathrm{CDCl}_{3}\right): \delta 2.78(\mathrm{~s}, 3 \mathrm{H}$, $\left.\mathrm{CH}_{3}\right), 3.22\left(\mathrm{t}, J 5.5 \mathrm{~Hz}, 2 \mathrm{H}, \mathrm{CH}_{2}\right), 3.27\left(\mathrm{~s}, 6 \mathrm{H}, \mathrm{CH}_{3}\right), 4.27(\mathrm{t}, J 5.3 \mathrm{~Hz}, 1 \mathrm{H}, \mathrm{CH}), 4.69(\mathrm{~s}, 1 \mathrm{H}, \mathrm{NH}) .{ }^{13} \mathrm{C} N M R\left(\mathrm{CDCl}_{3}\right): \delta$ 36.1, 42.4, 54.3, 103.4, 158.3. MALDI-TOF: $m / z 199[\mathrm{M}+\mathrm{Na}]^{+}$.

3-(2,2-Dimethoxyethyl)-1,1-diphenylurea (2b). Yellow oil, yield $2.35 \mathrm{~g}, 91 \% .{ }^{1} \mathrm{H}$ NMR $\left(\mathrm{CDCl}_{3}\right): \delta 3.39(\mathrm{~s}, 6 \mathrm{H}$, $\left.\mathrm{CH}_{3}\right), 4.43(\mathrm{t}, \mathrm{J} 5.4 \mathrm{~Hz}, 1 \mathrm{H}, \mathrm{CH}), 4.81(\mathrm{~s}, 1 \mathrm{H}, \mathrm{NH}), 7.25-7.21(\mathrm{~m}, 2 \mathrm{H}, \mathrm{ArH}), 7.39-7.27(\mathrm{~m}, 4 \mathrm{H}, \mathrm{ArH}), 7.35-7.37(\mathrm{~m}$, $4 \mathrm{H}, \mathrm{ArH}) .{ }^{13} \mathrm{C}$ NMR $\left(\mathrm{CDCl}_{3}\right): \delta 42.4,54.6,103.2,126.2,127.4,129.4,142.8,156.2$. MALDI-TOF: $\mathrm{m} / \mathrm{z} 323$ $[\mathrm{M}+\mathrm{Na}]^{+}$.

General method for the synthesis of diaryletanes $3 \mathbf{a}, \mathbf{b}$. To a mixture of phenol $(6.94 \mathrm{mmol})$, chloroform (10 $\mathrm{mL})$, and acetal $2(3.47 \mathrm{mmol})$, was added trifluoroacetic acid $(2 \mathrm{~mL})$. The reaction mixture was stirred for 24 hours at room temperature, the solvent was removed in vacuum, the residue was washed with diethyl ether and dried in vacuum.

3-[2,2-Bis(5-chloro-2,4-dihydroxyphenyl)ethyl]-1,1-dimethylurea (3a). Colorless solid. Yield $0.92 \mathrm{~g}, 66 \%$, m.p. 157-158 ${ }^{\circ} \mathrm{C}$. IR $\left(\mathrm{KBr}, v / \mathrm{cm}^{-1}\right): 1596,1627,2967,3089,3066,3283 .{ }^{1} \mathrm{H}$ NMR (DMSO- $\left.d_{6}\right): \delta 2.70\left(\mathrm{~s}, 6 \mathrm{H}, \mathrm{CH}_{3}\right), 3.41-$ $3.52\left(\mathrm{~m}, 2 \mathrm{H}, \mathrm{CH}_{2}\right), 4.48(\mathrm{t}, J 7.5 \mathrm{~Hz}, 1 \mathrm{H}, \mathrm{CH}), 6.19(\mathrm{~s}, 1 \mathrm{H}, \mathrm{NH}), 6.45(\mathrm{~s}, 2 \mathrm{H}, \mathrm{ArH}), 6.87(\mathrm{~s}, 2 \mathrm{H}, \mathrm{ArH}), 9.49(\mathrm{~s}, 2 \mathrm{H}$, 
$\mathrm{OH}), 9.72(\mathrm{~s}, 2 \mathrm{H}, \mathrm{OH}) .{ }^{13} \mathrm{C}$ NMR (DMSO- $\left.d_{6}\right): \delta 15.6,36.2,65.3,104.3,109.4,121.54,129.1,152.0,155.2,159.0$. Found (\%): $\mathrm{C}, 51.02 ; \mathrm{H}, 4.75 ; \mathrm{Cl}, 17.59 ; \mathrm{N}, 7.09$. Calc. for $\mathrm{C}_{17} \mathrm{H}_{18} \mathrm{Cl}_{2} \mathrm{~N}_{2} \mathrm{O}_{5}(\%): \mathrm{C}, 50.89 ; \mathrm{H}, 4.52 ; \mathrm{Cl}, 17.47 ; \mathrm{N}, 6.98$. MALDI-TOF: $m / z 423[\mathrm{M}+\mathrm{Na}]^{+}$.

3-[2,2-Bis(6-hydroxybenzo[d][1,3]dioxol-5-yl)ethyl]-1,1-diphenylurea (3b). Colorless solid. Yield $1.56 \mathrm{~g}, 88 \%$, m.p. $229-230{ }^{\circ} \mathrm{C}$. IR $\left(\mathrm{KBr}, v / \mathrm{cm}^{-1}\right): 1596,1626,2975,3058,3251 .{ }^{1} \mathrm{H}$ NMR (DMSO- $\left.d_{6}\right): \delta 3.43-3.57\left(\mathrm{~m}, 2 \mathrm{H}, \mathrm{CH}_{2}\right)$, $4.68(\mathrm{t}, J 8.1 \mathrm{~Hz}, 1 \mathrm{H}, \mathrm{CH}), 5.42(\mathrm{t}, J 5.5 \mathrm{~Hz}, 1 \mathrm{H}, \mathrm{NH}), 5.87\left(\mathrm{~d}, J 10.2 \mathrm{~Hz}, 4 \mathrm{H}, \mathrm{CH}_{2}\right), 6.44(\mathrm{~s}, 2 \mathrm{H}, \operatorname{ArH}), 6.67(\mathrm{~s}, 2 \mathrm{H}$, $\operatorname{ArH}), 7.00(\mathrm{~d}, J 7.9 \mathrm{~Hz}, 4 \mathrm{H}, \mathrm{ArH}), 7.15(\mathrm{t}, J 7.5 \mathrm{~Hz}, 2 \mathrm{H}, \mathrm{ArH}), 7.26(\mathrm{t}, J 7.7 \mathrm{~Hz}, 4 \mathrm{H}, \mathrm{ArH}), 9.01(\mathrm{~s}, 1 \mathrm{H}, \mathrm{OH}) .{ }^{13} \mathrm{C} \mathrm{NMR}$ (DMSO- $d_{6}$ ): $\delta 37.5,43.5,98.1,100.9,108.6,120.7,125.9,127.5,129.5,140.1,143.5,146.0,150.1,156.0$. Found (\%): C, 68.15; $\mathrm{H}, 4.90 ; \mathrm{N}, 5.21$. Calc. for $\mathrm{C}_{29} \mathrm{H}_{24} \mathrm{~N}_{2} \mathrm{O}_{7}$ (\%): C, 67.96; $\mathrm{H}, 4.72 ; \mathrm{N}, 5.47$. MALDI-TOF: $\mathrm{m} / z$ 513 $[\mathrm{M}+\mathrm{H}]^{+}, 535[\mathrm{M}+\mathrm{Na}]^{+}, 551[\mathrm{M}+\mathrm{K}]^{+}$.

General method for the synthesis of dibenzoxantenes 4a-c. To a mixture of naphthol (2.95 mmol), chloroform $(5 \mathrm{~mL})$, and acetal $2(1.47 \mathrm{mmol})$, was added trifluoroacetic acid $(2 \mathrm{~mL})$. The reaction mixture was stirred for 24 hours at room temperature, the solvent was removed in vacuum, the residue was washed with diethyl ether and dried in vacuum.

3-[(14H-dibenzo[a,j]xanthen-14-yl)methyl]-1,1-dimethylurea (4a). Colorless solid. Yield 0.49 g, 87\%, m.p. 250-251 ${ }^{\circ} \mathrm{C}$. IR $\left(\mathrm{KBr}, \mathrm{v} / \mathrm{cm}^{-1}\right): 1593,1676,2975,3013,3374 .{ }^{1} \mathrm{H}$ NMR (DMSO-d 6 ): $\delta 2.57\left(\mathrm{~s}, 6 \mathrm{H}, \mathrm{CH}_{3}\right), 3.27(\mathrm{t}, J$ $\left.6.2 \mathrm{~Hz}, 2 \mathrm{H}, \mathrm{CH}_{2}\right), 5.79(\mathrm{t}, J 6.8 \mathrm{~Hz}, 1 \mathrm{H}, \mathrm{CH}), 6.28(\mathrm{t}, J 5.5 \mathrm{~Hz}, 1 \mathrm{H}, \mathrm{NH}), 7.47(\mathrm{~d}, J 8.9 \mathrm{~Hz}, 2 \mathrm{H}, \mathrm{ArH}), 7.49(\mathrm{t}, J 7.0 \mathrm{~Hz}$, $2 \mathrm{H}, \mathrm{ArH}), 7.61(\mathrm{t}, J 7.1 \mathrm{~Hz}, 2 \mathrm{H}, \mathrm{ArH}), 7.91(\mathrm{~d}, J 8.9 \mathrm{~Hz}, 2 \mathrm{H}, \mathrm{ArH}), 7.94(\mathrm{~d}, J 7.9 \mathrm{~Hz}, 2 \mathrm{H}, \mathrm{ArH}), 8.51(\mathrm{~d}, J 8.5 \mathrm{~Hz}, 2 \mathrm{H}$, ArH). ${ }^{13} \mathrm{C}$ NMR (DMSO- $d_{6}$ ): $\delta 30.9,36.2,47.6,117.2,117.7,123.5,124.8,127.0,128.8,129.0,131.0,132.5$, 150.1, 158.9. Found (\%): C, 78.63; $\mathrm{H}, 5.94 ; \mathrm{N}, 7.50$. Calc. for $\mathrm{C}_{25} \mathrm{H}_{22} \mathrm{~N}_{2} \mathrm{O}_{2}$ (\%): C, 78.51; $\mathrm{H}, 5.80 ; \mathrm{N}, 7.32$. MALDITOF: $m / z 382[\mathrm{M}+\mathrm{H}]^{+}$.

3-[(2,12-Dihydroxy-14H-dibenzo[ $a, j] x a n t h e n-14-y l) m e t h y l]-1,1-d i m e t h y l u r e a ~(4 b)$. Colorless solid. Yield 0.43 g, 70\%, m.p. $>250{ }^{\circ} \mathrm{C}$. IR $\left(\mathrm{KBr}, v / \mathrm{cm}^{-1}\right): 1595,1680,2875,3085,3319 .{ }^{1} \mathrm{H}$ NMR (DMSO-d 6$): \delta 2.58\left(\mathrm{~s}, 6 \mathrm{H}, \mathrm{CH}_{3}\right)$, $3.23\left(\mathrm{t}, J 6.1 \mathrm{~Hz}, 2 \mathrm{H}, \mathrm{CH}_{2}\right), 5.40(\mathrm{t}, J 6.7 \mathrm{~Hz}, 1 \mathrm{H}, \mathrm{CH}), 6.13(\mathrm{t}, J 5.6 \mathrm{~Hz}, 1 \mathrm{H}, \mathrm{NH}), 7.07$ (dd, J 8.7, 2.2 Hz, 2H, ArH), $7.18(\mathrm{~d}, J 8.8 \mathrm{~Hz}, 2 \mathrm{H}, \mathrm{ArH}), 7.69(\mathrm{~d}, J 1.9 \mathrm{~Hz}, 2 \mathrm{H}, \mathrm{ArH}), 7.73(\mathrm{~d}, J 8.9 \mathrm{~Hz}, 2 \mathrm{H}, \mathrm{ArH}), 7.76(\mathrm{~d}, J 8.9 \mathrm{~Hz}, 2 \mathrm{H}, \mathrm{ArH}) .{ }^{13} \mathrm{C}$ NMR (DMSO- $\left.d_{6}\right): \delta 31.3,36.2,46.8,105.7,114.3,115.9,117.0,125.5,128.6,130.3,134.4,150.6,156.8,158.9$. Found (\%): C, 72.57; H, 5.49; N, 6.52. Calc. for $\mathrm{C}_{25} \mathrm{H}_{22} \mathrm{~N}_{2} \mathrm{O}_{4}$ (\%): C, 72.45; H, 5.35; N, 6.76. MALDI-TOF: $m / z 415$ $[\mathrm{M}+\mathrm{H}]^{+}, 437[\mathrm{M}+\mathrm{Na}]^{+}$.

3-[(14H-Dibenzo[a,j]xanthen-14-yl)methyl]-1,1-diphenylurea (4c). Colorless solid. Yield $0.59 \mathrm{~g}, 79 \%, \mathrm{~m} . \mathrm{p}$. $>250{ }^{\circ} \mathrm{C}$. IR $\left(\mathrm{KBr}, v / \mathrm{cm}^{-1}\right): 1591,1676,2921,3059,3414 .{ }^{1} \mathrm{H}$ NMR (DMSO-d 6 ): $\delta 3.40\left(\mathrm{t}, J 6.0 \mathrm{~Hz}, 2 \mathrm{H}, \mathrm{CH}_{2}\right), 5.74$ $(\mathrm{t}, J 5.6 \mathrm{~Hz}, 1 \mathrm{H}, \mathrm{CH}), 5.81(\mathrm{t}, J 6.4 \mathrm{~Hz}, 1 \mathrm{H}, \mathrm{NH}), 6.79(\mathrm{~d}, J 7.5 \mathrm{~Hz}, 4 \mathrm{H}, \mathrm{ArH}), 7.13(\mathrm{t}, J 7.4 \mathrm{~Hz}, 2 \mathrm{H}, \operatorname{ArH}), 7.23(\mathrm{t}, J 7.8$ $\mathrm{Hz}, 4 \mathrm{H}, \mathrm{ArH}), 7.44(\mathrm{~d}, J 8.8 \mathrm{~Hz}, 2 \mathrm{H}, \mathrm{ArH}), 7.51(\mathrm{t}, J 7.3 \mathrm{~Hz}, 2 \mathrm{H}, \mathrm{ArH}), 7.66(\mathrm{t}, J 7.2 \mathrm{~Hz}, 2 \mathrm{H}, \mathrm{ArH}), 7.90(\mathrm{~d}, J 8.8 \mathrm{~Hz}$, $2 \mathrm{H}, \mathrm{ArH}), 7.97(\mathrm{~d}, J 8.0 \mathrm{~Hz}, 2 \mathrm{H}, \mathrm{ArH}), 8.57(\mathrm{~d}, J 8.5 \mathrm{~Hz}, 2 \mathrm{H}, \mathrm{ArH}) .{ }^{13} \mathrm{C} N M R\left(D M S O-d_{6}\right): \delta 46.6,116.6,117.8$, 123.5, 124.9, 126.2, 127.3, 128.0, 129.0, 129.1, 129.5, 131.0, 132.3, 143.2, 150.2, 156.1. Found (\%): C, 83.11; $\mathrm{H}, 4.99 ; \mathrm{N}, 5.74$. Calc. for $\mathrm{C}_{35} \mathrm{H}_{26} \mathrm{~N}_{2} \mathrm{O}_{2}$ (\%): C, 82.98; $\mathrm{H}, 5.17 ; \mathrm{N}, 5.53$. MALDI-TOF: $\mathrm{m} / z 507$ [M+H] ${ }^{+}, 529[\mathrm{M}+\mathrm{Na}]^{+}$, $545[\mathrm{M}+\mathrm{K}]^{+}$.

3-[(2,12-Dihydroxy-14H-dibenzo[a,j]xanthen-14-yl)methyl]-1,1-diphenylurea (4d). Colorless solid. Yield 0.54 g, 68\%, m.p. $222{ }^{\circ} \mathrm{C}$. IR (KBr, v/cm ${ }^{-1}$ ): 1593, 1675, 2986, 3078, 3446. ${ }^{1} \mathrm{H}$ NMR (DMSO- $\left.d_{6}\right): \delta 3.21-3.35$ (m, 2H, $\mathrm{CH}_{2}$ ), $5.42(\mathrm{t}, J 6.0 \mathrm{~Hz}, 1 \mathrm{H}, \mathrm{CH}), 5.49(\mathrm{t}, J 5.7 \mathrm{~Hz}, 1 \mathrm{H}, \mathrm{NH}), 6.94(\mathrm{~d}, J 7.3 \mathrm{~Hz}, 4 \mathrm{H}, \mathrm{ArH}), 7.13-7.08(\mathrm{~m}, 4 \mathrm{H}, \mathrm{ArH})$, $7.16(\mathrm{~d}, J 9.0 \mathrm{~Hz}, 2 \mathrm{H}, \mathrm{ArH}), 7.21(\mathrm{t}, J 7.5 \mathrm{~Hz}, 4 \mathrm{H}, \mathrm{ArH}), 7.76-7.70(\mathrm{~m}, 4 \mathrm{H}, \mathrm{ArH}), 7.80(\mathrm{~d}, J 8.8 \mathrm{~Hz}, 2 \mathrm{H}, \mathrm{ArH}) .{ }^{13} \mathrm{C}$ NMR (DMSO- $\left.d_{6}\right): \delta 31.4,45.9,105.6,114.3,115.1,117.1,125.5,126.3,128.3,128.7,129.4,130.5,134.0$, 143.3, 150.6, 156.2, 157.0. Found (\%): C, 77.84; $\mathrm{H}, 4.99 ; \mathrm{N}, 5.34$. Calc. for $\mathrm{C}_{35} \mathrm{H}_{26} \mathrm{~N}_{2} \mathrm{O}_{4}(\%): \mathrm{C}, 78.05 ; \mathrm{H}, 4.87 ; \mathrm{N}$, 5.20. MALDI-TOF: $m / z 539[\mathrm{M}+\mathrm{H}]^{+}, 561[\mathrm{M}+\mathrm{Na}]^{+}, 577[\mathrm{M}+\mathrm{K}]^{+}$. 


\section{Acknowledgements}

This study was performed with financial support from the scholarship of the President of the Russian Federation (No. CП-943.2019.4). X-Ray studies were funded by Russian Science Foundation (Grant No. 19-1300238). The authors are grateful to the Assigned Spectral-Analytical Center of FRC Kazan Scientific Center of RAS for technical assistance in research. The x-ray measurements were performed using shared experimental facilities supported by IGIC RAS state assignment.

\section{Supplementary Material}

Copies of ${ }^{1} \mathrm{H}$ and ${ }^{13} \mathrm{C}$ NMR spectra of all products and details of the crystallographic studies can be found at Supporting Information.

\section{References}

1. Beck, T. N.; Lloyd, D.; Kuskovsky, R.; Minah, J.; Arora, K.; Plotkin, B. J.; Green, J. M.; Boshoff, H. I.; Barry, C.; Deschamps, J.; Konaklieva, M. I. Bioorg. Med. Chem. 2015, 23, 632.

https://doi.org/10.1016/j.bmc.2014.11.025

2. Dobrikov, G. M.; Valcheva, V.; Nikolova, Y.; Ugrinova, I.; Pasheva, E.; Dimitrov, V. Eur. J. Med. Chem. 2013, $63,468$.

https://doi.org/10.1016/i.ejmech.2013.02.034

3. Wang, B.; Pachaiyappan, B.; Gruber, J. D.; Schmidt, M. G.; Zhang, Y.-M.; Woster, P. M. J. Med. Chem. 2016, $59,3140$. https://doi.org/10.1021/acs.jmedchem.5b01912

4. Nowotarski, S. L.; Pachaiyappan, B.; Holshouser, S. L.; Kutz, C. J.; Li, Y.; Huang, Y.; Sharma, S. K.; Casero, R. A.; Woster, P. M. Bioorg. Med. Chem. 2015, 23, 1601.

https://doi.org/10.1016/j.bmc.2015.01.049

5. Lainé, D. I.; Wan, Z.; Yan, H.; Zhu, C.; Xie, H.; Fu, W.; Busch-Petersen, J.; Neipp, C.; Davis, R.; Widdowson, K. L.; Blaney, F. E.; Foley, J.; Bacon, A. M.; Webb, E. F.; Luttmann, M. A.; Burman, M.; Sarau, H. M.; Salmon, M.; Palovich, M. R.; Belmonte, K. J. Med. Chem. 2009, 52, 5241.

\section{https://doi.org/10.1021/jm900736e}

6. De Lucca, G. V.; Kim, U. T.; Johnson, C.; Vargo, B. J.; Welch, P. K.; Covington, M.; Davies, P.; Solomon, K. A.; Newton, R. C.; Trainor, G. L.; Decicco, C. P.; Ko, S. S. J. Med. Chem. 2002, 45, 3794.

https://doi.org/10.1021/jm0201767

7. Verlinden, B. K.; Niemand, J.; Snyman, J.; Sharma, S. K.; Beattie, R. J.; Woster, P. M.; Birkholtz, L.-M. J. Med. Chem. 2011, 54, 6624.

https://doi.org/10.1021/jm200463z

8. De Vries, V. G.; Bloom, J. D.; Dutia, M. D.; Katocs, A. S.; Largis, E. E. J. Med. Chem. 1989, 32, 2318. https://doi.org/10.1021/jm00130a016

9. Abdulla, R. F.; Brinkmeyer, R. S. Tetrahedron 1979, 35, 1675. 
https://doi.org/10.1016/0040-4020(79)88001-1

10. Smolobochkin, A. V.; Gazizov, A. S.; Burilov, A. R.; Pudovik, M. A. Chem. Heterocycl. Compd. 2016, 52, 753. https://doi.org/10.1007/s10593-016-1960-1

11. Gazizov, A. S.; Burilov, A. R.; Pudovik, M. A.; Sinyashin, O. G. Russ. Chem. Rev. 2017, 86, 75. https://doi.org/10.1070/RCR4622

12. Granik, V. G.; Zhidkova, A. M.; Glushkov, R. G. Russ. Chem. Rev. 1977, 46, 361. https://doi.org/10.1070/RC1977v046n04ABEH002137

13. Yamauchi, T.; Hagiwara, S.; Higashiyama, K. J. Org. Chem. 2008, 73, 9784. https://doi.org/10.1021/jo801700f

14. Amat, M.; Elias, V.; Llor, N.; Subrizi, F.; Molins, E.; Bosch, J. Eur. J. Org. Chem. 2010, 4017. https://doi.org/10.1002/ejoc.201000473

15. Louafi, F.; Moreau, J.; Shahane, S.; Golhen, S.; Roisnel, T.; Sinbandhit, S.; Hurvois, J.-P. J. Org. Chem. 2011, $76,9720$.

https://doi.org/10.1021/jo2017982

16. La Regina, G.; Famiglini, V.; Passacantilli, S.; Pelliccia, S.; Punzi, P.; Silvestri, R. Synthesis (Stuttg). 2014, 46, 2093.

https://doi.org/10.1055/s-0033-1339155

17. Kuehne, M. E.; He, L.; Jokiel, P. A.; Pace, C. J.; Fleck, M. W.; Maisonneuve, I. M.; Glick, S. D.; Bidlack, J. M. J. Med. Chem. 2003, 46, 2716.

https://doi.org/10.1021/jm0205620

18. Cioc, R. C.; Ruijter, E.; Orru, R. V. A. Green Chem. 2014, 16, 2958.

https://doi.org/10.1039/C4GC00013G

19. Smolobochkin, A. V.; Rizbayeva, T. S.; Gazizov, A. S.; Voronina, J. K.; Dobrynin, A. B.; Gildebrant, A. V.; Strelnik, A. G.; Sazykin, I. S.; Burilov, A. R.; Pudovik, M. A.; Sazykina, M. A. Eur. J. Org. Chem. 2019, 5709. https://doi.org/10.1002/ejoc.201900868

20. Khakimov, M. S.; Gazizov, A. S.; Burilov, A. R.; Pudovik, M. A.; Konovalov, A. I. Russ. J. Gen. Chem. 2009, 79, 1163.

https://doi.org/10.1134/S107036320906022X

21. Gazizov, A. S.; Khakimov, M. S.; Burilov, A. R.; Pudovik, M. A.; Krivolapov, D. B.; Litvinov, I. A.; Konovalov, A. I. Russ. Chem. Bull. 2009, 58, 238.

https://doi.org/10.1007/s11172-009-0036-0

22. Burilov, A. R.; Gazizov, A. S.; Khakimov, M. S.; Kharitonova, N. I. I.; Pudovik, M. A.; Konovalov, A. I. Russ. J. Gen. Chem. 2008, 78, 2411. https://doi.org/10.1134/S1070363208120220

23. Smolobochkin, A. V.; Gazizov, A. S.; Burilov, A. R.; Pudovik, M. A. Synth. Commun. 2018, 48, 2545. https://doi.org/10.1080/00397911.2018.1512000

24. Gazizov, A. S.; Smolobochkin, A. V.; Anikina, E. A.; Strelnik, A. G.; Burilov, A. R.; Pudovik, M. A. Synlett 2018, $29,467$.

https://doi.org/10.1055/s-0036-1590954

25. Gazizov, A. S.; Smolobochkin, A. V.; Voronina, J. K.; Burilov, A. R.; Pudovik, M. A. Tetrahedron 2015, 71, 445.

https://doi.org/10.1016/i.tet.2014.12.011

26. Sheldrick, G. M. SHELXTL v.6.12, Structure Determination Software Suite, Bruker AXS, Madison, WI, USA, 2000. 
27. Dolomanov, O. V.; Bourhis, L. J.; Gildea, R. J.; Howard, J. A. K.; Puschmann, H. J. Appl. Crystallogr. 2009, 42, 339-341.

https://doi.org/10.1107/s0021889808042726

28. Klimova, V. A. Basic Micromethods for Analysis of Organic Compounds [in Russian]; Khimiya: Moscow, 1975, p. 104. 